\section{Guiding New Generations for Decades}

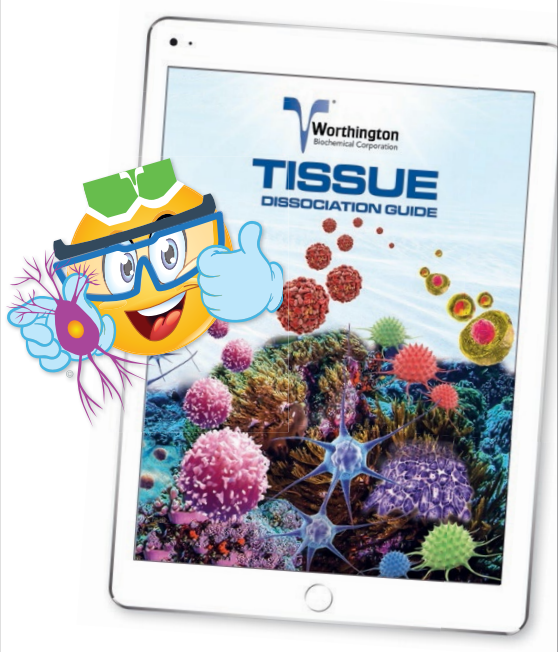

Worthington is supporting life science research and discovery, and we have done so for over 70 years.

Authoring technical manuals and guides both in print and digital formats. And, we are cited in thousands of respected scientific journals across the globe.

\section{Sharing Our Enzyme Expertise}

\section{Order your free copy of the}

NEW Tissue Dissociation Guide,

18th Edition. Simply go to:

\section{Worthington-Biochem.com/ TissueDissociation/default.html}

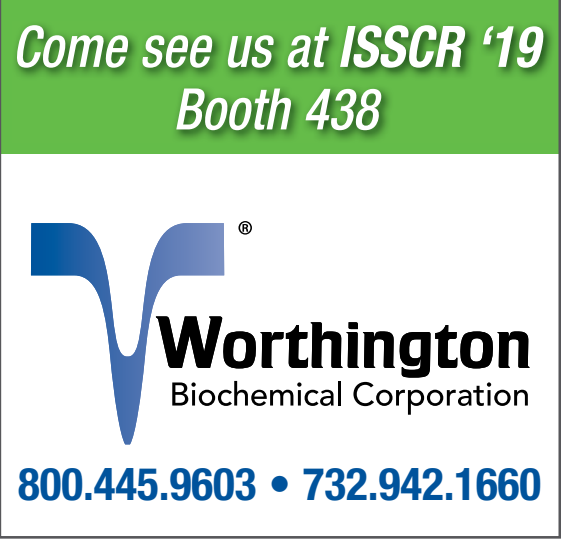

\title{
Protein translation inside synthetic membraneless organelles
}

Phase separation in combination with spatial targeting allows for selective translation of mRNA within synthetic membraneless organelles.

D uring the past years, the concept of intracellular membraneless compartments formed through liquid-liquid phase separation has entered a new 'phase' in cell biology owing to its critical role in cellular functions from gene regulation to cell signaling. Researchers now are starting to take advantage of the properties of membraneless compartments to compartmentalize functions in living cells.

These membraneless compartments allow one to dynamically and locally concentrate molecules and thus spatiotemporally regulate reactions inside living cells. Edward Lemke and colleagues at the European Molecular Biology Laboratory (EMBL), Johannes Gutenberg University, and IMB Mainz propose to generate a synthetic membraneless compartment, a membraneless organelle, to host orthogonal protein translation in eukaryotic cells. "As intracellular phase separation is one way cells naturally achieve both dynamic exchange with the cytoplasm and a high local concentration, we thought that we could use these properties to build our organelle," says Lemke, whose group has a strong interest in chemical biology and protein-engineeringenabled labeling technologies.

In light of genetic code expansion (GCE) technology, researchers have been able to incorporate noncanonical amino acids (ncAAs) into proteins site-specifically by reallocating specific codons. However, GCE can suffer from off-target ncAA incorporation and potential toxicity caused by genome-wide GCE.

To reduce interference, Lemke proposes to "simultaneously operate two genetic codes in one cell: the canonical genetic code for all housekeeping proteins; as well as the expanded genetic code exclusively for our protein of interest." The key for the design of an orthogonally translating (OT) organelle is to specifically confine the mRNA of interest, as well as the suppressor tRNA and tRNA synthetase pair, within the organelle and spatially separate them from the rest of the cytoplasm. To this end, Lemke and colleagues engineered membraneless organelles by fusing the suppressor tRNA

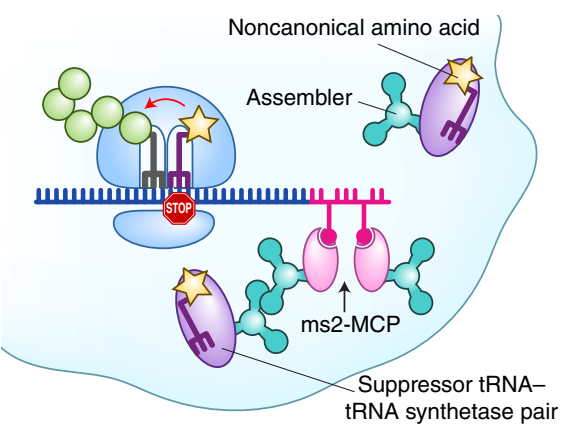

Synthetic membraneless organelles enable selective translation of mRNA. Credit: Marina Corral Spence/Springer Nature

synthetase and an mRNA-binding domain with proteins containing intrinsically disordered domains that are known to form droplet-like structures.

However, Lemke comments, "we realized that adding a spatial separation component, like kinesin motor proteins, does further locally constrain the components of our organelle and thereby really helps to increase selectivity." Thus, they fused a truncated kinesin to the phase-separated species in order to achieve spatial targeting to microtubule-plus ends. The combination of phase separation (P system) and kinesin motor proteins ( $\mathrm{K}$ system) can generate micrometer-sized organelle-like structures in the cytoplasm, which can serve as OT organelles with selectivity and efficiency.

Beyond protein translation, Lemke remarks that "our work underlines that membrane-less organelles can perform complex and specific tasks, despite being constituted from a few components. We can thus also learn a bit on how naturally occurring membraneless organelles can and might function."

\section{Lei Tang}

Published online: 30 May 2019

https://doi.org/10.1038/s41592-019-0439-2

\section{Research papers}

Reinkemeier, C. D. et al. Designer membraneless organelles enable codon reassignment of selected mRNAs in eukaryotes. Science 363, 1415 (2019). 